\title{
Proton Structure Measurements from HERA to LHC
}

\author{
Vladimir Chekelian (Shekelyan) ${ }^{\mathrm{a}}$ \\ ${ }^{a}$ MPI for Physics, Munich \\ On behalf of the HI and ZEUS collaborations
}

\begin{abstract}
The inclusive ep neutral and charged current deep-inelastic scattering cross sections are measured in the H1 and ZEUS experiments at HERA, with an electron beam energy of $27.6 \mathrm{GeV}$ and a nominal proton beam energy of $920 \mathrm{GeV}$ and reduced proton beam energies of 575 and $460 \mathrm{GeV}$. The data span six orders of magnitude in negative fourmomentum-transfer squared, $Q^{2}$, and in Bjorken x. These data are used to determine the proton structure functions and to perform analyses in the QCD framework. The parton distribution functions of the proton, determined in QCD fits, are used for cross section calculations of various processes and for comparisons to data at the LHC.
\end{abstract}

Keywords: HERA, DIS, neutral and charged currents, structure functions, QCD, parton distribution functions

\section{Introduction}

The first electron-proton collider HERA operated over 15 years from 1992 to 2007. The inclusive neutral current (NC) and charged current (CC) deep-inelastic scattering (DIS) at HERA has been central to the exploration of proton structure and quark-gluon interaction dynamics as prescribed in perturbative Quantum Chromodynamics (QCD).

At HERA electrons and positrons of $27.6 \mathrm{GeV}$ collided with protons of $920 \mathrm{GeV}$ (820 GeV until 1997) corresponding to an $e p$ centre-of-mass energy of $319 \mathrm{GeV}$. The operation of HERA proceeded in two phases, HERA I (1992-2000) with an unpolarised and HERA II (2003-2007) with a longitudinally polarised lepton beam. At the end of the HERA data taking, special runs with reduced proton beam energies of 460 and $575 \mathrm{GeV}$ were taken for dedicated $F_{L}$ measurement. The two $e p$ interaction regions were instrumented with the multi-purpose detectors of the $\mathrm{H} 1$ and ZEUS collider experiments.

The inclusive $\mathrm{NC}$ and $\mathrm{CC}$ data from HERA are used to determine the proton structure functions and the partonic structure of the proton. The modern parton distribution functions (PDFs) are determined in QCD fits with the HERA data as an obligatory input to the fit. The PDFs are used for the calculation of cross sections of various processes and for comparisons to data at the LHC. The HERA data are analysed in the QCD framework to provide the HERAPDF sets of PDFs. On the basis of the HERAPDF environment an open access plat-

Preprint submitted to Nuclear Physics B form HERAFitter has been developed and is used by the LHC experiments.

\section{Neutral and Charged Current Cross Sections}

The NC cross section for $e^{ \pm} p$ scattering can be expressed in a reduced form in terms of generalised proton structure functions $\tilde{F}$ as

$$
\begin{array}{r}
\tilde{\sigma}_{N C}^{ \pm}\left(x, Q^{2}\right)=\frac{\mathrm{d}^{2} \sigma_{N C}^{e^{ \pm} p}}{\mathrm{~d} x \mathrm{~d} Q^{2}} \frac{x Q^{4}}{2 \pi \alpha^{2}} \frac{1}{Y_{+}}= \\
\tilde{F}_{2}\left(x, Q^{2}\right) \mp \frac{Y_{-}}{Y_{+}} x \tilde{F}_{3}\left(x, Q^{2}\right)-\frac{y^{2}}{Y_{+}} \tilde{F}_{L}\left(x, Q^{2}\right),
\end{array}
$$

where $\alpha$ is the fine structure constant, $x$ is the Bjorken scaling variable, $Q^{2}$ is the negative four-momentumtransfer squared, and $y$ characterises the inelasticity of the interaction with $Y_{ \pm}=1 \pm\left(1-y^{2}\right)$.

The generalised structure functions $\tilde{F}_{2,3}$ may be written as linear combinations of the structure functions $F_{2}$, $F_{2,3}^{\gamma Z}$ and $F_{2,3}^{Z}$, which contain information on QCD parton dynamics as well as on the electroweak couplings of the quarks to the neutral vector bosons. The $F_{2,3}^{\gamma Z}$ and $F_{2,3}^{Z}$ contributions to $\tilde{F}_{2,3}$ depend on the longitudinal polarisation of the lepton beam $P_{e}$. The electromagnetic structure function $F_{2}$ is associated with pure photon exchange, $F_{2,3}^{\gamma Z}$ correspond to photon- $Z$ interference, and $F_{2,3}^{Z}$ describe pure $Z$-exchange. In the quark-parton model $(\mathrm{QPM}) \tilde{F}_{L} \equiv 0 . \tilde{F}_{L}$ contributes only at high $y$ and is expected in QCD to be negligible at large $x$ and $Q^{2}$.

September 20, 2012 
The CC $e^{ \pm} p$ cross section of longitudinally polarised leptons and unpolarised protons can be expressed in the reduced form as

$$
\begin{gathered}
\tilde{\sigma}_{\mathrm{CC}}^{ \pm}\left(x, Q^{2}\right) \equiv \frac{4 \pi x}{G_{F}^{2}}\left[\frac{M_{W}^{2}+Q^{2}}{M_{W}^{2}}\right]^{2} \frac{\mathrm{d}^{2} \sigma_{\mathrm{CC}}^{e^{ \pm} p}}{\mathrm{~d} x \mathrm{~d} Q^{2}}= \\
\left(1 \pm P_{e}\right)\left(Y_{+} W_{2}^{ \pm} \mp Y_{-} x W_{3}^{ \pm}-y^{2} W_{L}^{ \pm}\right),
\end{gathered}
$$

where $G_{F}$ is the Fermi constant defined using the weak boson masses $M_{W}, M_{Z}$. In the QPM $W_{L}^{ \pm} \equiv 0$, and the structure functions $W_{2}^{ \pm}$and $x W_{3}^{ \pm}$are expressed below the $b$ quark mass threshold as the flavour dependent sum and difference of the quark and anti-quark momentum distributions:

$$
\begin{aligned}
W_{2}^{-}=x(u+c+\bar{d}+\bar{s}), & W_{2}^{+}=x(\bar{u}+\bar{c}+d+s), \\
x W_{3}^{-}=x(u+c-\bar{d}-\bar{s}), & x W_{3}^{+}=x(d+s-\bar{u}-\bar{c}) .
\end{aligned}
$$

\section{HERA inclusive data}

The general purpose detectors of $\mathrm{H} 1$ and ZEUS have a nearly $4 \pi$ coverage. They were designed to study the full range of $e p$ physics at HERA. The larger proton beam energy led to asymmetric instrumentation in the proton and the electron beam directions. The liquid argon (LAr) and spagetti-type (SpaCal) calorimeters in $\mathrm{H} 1$ and the depleted uranium-scintillator $(\mathrm{U} / \mathrm{Sc})$ calorimeter in ZEUS ensured measurements of the scattered electron and the hadronic final state particles. Drift chambers in the central region, placed in a solenoidal magnetic field, were used to measure tracks related to the charged particles originated from the interaction point. The chambers were supplemented by silicon tracker around the interaction region. The calorimeters were surrounded by an instrumented iron yoke used as a tail catcher and for muon identification. The ability to measure the complete final state provided a possibility to reconstruct event kinematics in different ways. Depending on the phase space, the measurements are done applying methods with the best performance. Luminosity was measured using the Bethe-Heitler process of QED bremsstrahlung $e p \rightarrow e p \gamma$ and the elastic QED compton process [1].

After the luminosity upgrade of the HERA collider in 2003, the lepton beam was longitudinally polarised. Each of the two experiments, H1 and ZEUS, collected a total integrated luminosity of $\approx 0.5 \mathrm{fb}^{-1}$, about equally shared between positive and negative polarities and positive and negative longitudinal polarisations of the lepton beam. The H1 and ZEUS inclusive cross section data comprise many data sets, which were obtained using different detector components and different running conditions.

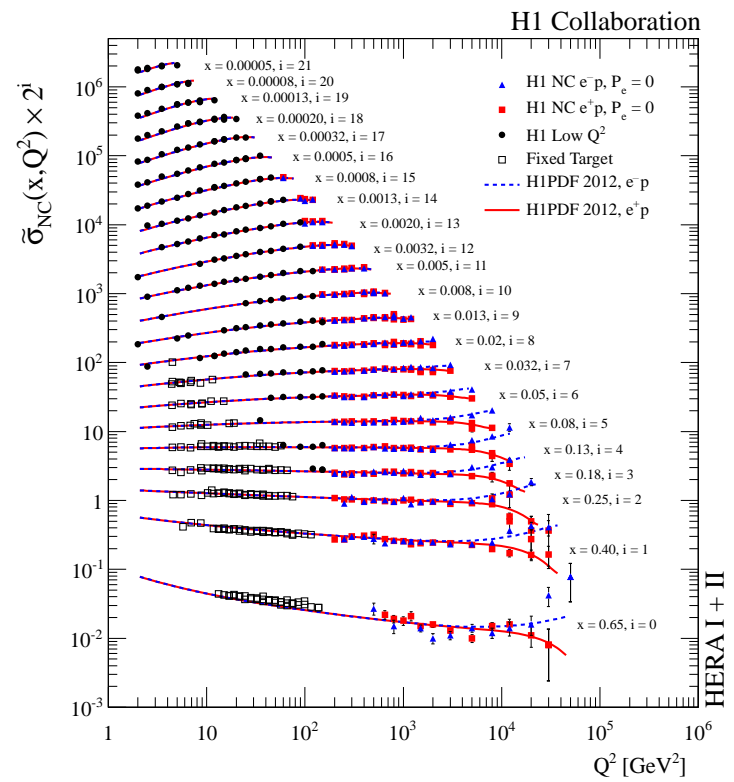

Figure 1: The NC $e^{-} p$ and $e^{+} p$ reduced cross sections $\tilde{\sigma}_{N C}$ measured by $\mathrm{H} 1$ (solid symbols) using HERA I+II data are shown for various fixed $x$ as a function of $Q^{2}$. The curves show the corresponding expectations from H1PDF 2012 [5]. The fixed target data from BCDMS are also shown (open squares).

\subsection{Neutral currents}

The measurements of inclusive $\mathrm{NC}$ reactions at HERA extend over a wide range in $x$ and $Q^{2}$ : from very low $x=6 \cdot 10^{-7}$ and $Q^{2}=0.045 \mathrm{GeV}^{2}$ to $x=0.65$ and $Q^{2}=50000 \mathrm{GeV}^{2}$.

The polar angle of the scattered electron is related to the value of $Q^{2}$. Small scattering angles correspond to low $Q^{2}$. In the low $Q^{2}$ region, $Q^{2} \leq 100 \mathrm{GeV}^{2}$, the electron scatters in the $\mathrm{H} 1$ experiment into the $\mathrm{SpaCal}$ backward calorimeter. The high $Q^{2}$ domain is covered by the measurements of the scattered electron in the LAr calorimeter. In ZEUS the U/Sc calorimeter covers both small and large scattering angles.

The inclusive cross section measurement program in the low $Q^{2}$ domain was completed using the HERA I data [2]. An accuracy of the measurements of about $2 \%$ is obtained at $Q^{2} \approx 10 \mathrm{GeV}^{2}$. During the luminosity upgrade of HERA, strong focusing magnets were placed near the interaction point inside the $\mathrm{H} 1$ and ZEUS detectors. This caused restrictions of the detectors acceptances at low $Q^{2}$.

At high $Q^{2}$ the statistical precision of the HERA I measurements [2] is limited. The HERA II data improve statistics by a factor of three for $e^{+} p$ and by a factor of ten for $e^{-} p$ data $[3,4]$. The longitudinal polarisation of 
the lepton beam after the upgrade allowed for investigation of polarisation effects at high $Q^{2}$.

Recently $\mathrm{H} 1$ and ZEUS completed the inclusive $e p$ measurements at HERA by publishing the $e^{ \pm} p$ NC and CC cross sections at high $Q^{2}$ from HERA II (H1) [5] and the $e^{+} p$ NC cross section from the years 2006-2007 (ZEUS) [6]. These measurements have two times better precision than the HERA I results, reaching $\approx 1.5 \%$ accuracy in the region of $Q^{2}$ of a few hundred $\mathrm{GeV}^{2}$. The reduced NC HERA I+II cross section measurements by $\mathrm{H} 1$ are shown in Figure 1. These cross sections are equivalent to the electromagnetic proton structure function $F_{2}$ in the bulk of the phase space. The difference between the $e^{-} p$ and $e^{+} p$ data at high $Q^{2}$ is due to $x \tilde{F}_{3}$ entering the cross section with opposite sign, see equation 1 . This difference is exploited to measure $x F_{3}^{\gamma Z}[5,6]$, which is sensitive to valence quarks. The dependence of the HERA II cross sections measurements on the longitudinal polarisation of the lepton beam is used to measure the parity violating structure function $F_{2}^{\gamma Z}$ for the first time [5]. The longitudinal structure function $F_{L}$ is obtained at HERA $[7,8]$ in a model independent way using measurements at fixed $x$ and $Q^{2}$ but different $y$. This is achieved using running periods with reduced $(460 \mathrm{GeV}$ and $575 \mathrm{GeV})$ and nominal $(920 \mathrm{GeV})$ proton beam energies.

\subsection{Charged currents}

In the $\mathrm{CC}$ process the outgoing neutrino escapes detection. These interaction are recognised by the momentum imbalance of the final state particles in the transverse plane to the beam direction, i.e. $p_{t}^{\text {miss }} \geq 12 \mathrm{GeV}$.

The inclusive CC cross sections are measured [2, 3, 5] in the range $1.3 \cdot 10^{-2} \leq x \leq 0.65$ and $300 \leq Q^{2} \leq$ $30000 \mathrm{GeV}^{2}$. The $e^{+} p$ and $e^{-} p \mathrm{CC}$ data are sensitive to different combinations of the light flavours in the proton. For example the $e^{+} p$ cross sections are governed by the down-quark density of the proton. This allows for a flavour decomposition of the content of the proton in the QCD analysis of the HERA data.

The polarisation effects in CC are studied at HERA II using the total $\mathrm{CC}$ cross section defined in the region $Q^{2}>400 \mathrm{GeV}^{2}$ and $y<0.9$. The Standard Model predicts that, in the absence of right-handed charged currents, the $e^{-} p\left(e^{+} p\right)$ CC cross section is directly proportional to the fraction of right-handed electrons (lefthanded positrons) in the beam. The measurements exhibit the expected linear dependences as a function of the polarisation $P_{e}$.

\section{Combination of the $\mathrm{H} 1$ and ZEUS inclusive cross section data}

All HERA I inclusive NC and CC cross sections published by $\mathrm{H} 1$ and ZEUS were combined in one simultaneous minimization [2]. The resulting shifts of the correlated systematic uncertainties are propagated to both $\mathrm{NC}$ and $\mathrm{CC}$ data such that one coherent data set is obtained. Since H1 and ZEUS have employed different experimental techniques, different detectors and methods of kinematic reconstruction, the averaging leads to a significantly reduced correlated systematic uncertainty. This reduction propagates to all averaged points, including also those which are based solely on the measurement from one experiment. The total uncertainty of the combined data set reaches $1 \%$ for NC scattering in the best measured region, $20<Q^{2}<100 \mathrm{GeV}^{2}$.

The averaging is extended to include preliminary H1 [4] and published ZEUS [3] measurements from the HERA II running period. The inclusion of the large HERA II data set leads to an improved uncertainty especially at high $Q^{2}$ and $x$ [9].

With the recent publications of the final $\mathrm{H} 1$ and ZEUS results at high $Q^{2}$ from HERA II the next step to come is a combination of the entire HERA I+II inclusive data into one complete and coherent set of the inclusive $\mathrm{NC}$ and $\mathrm{CC}$ cross section data at HERA.

\section{HERAPDF and HERAFitter}

H1 and ZEUS performed a series of next-to-leading order (NLO) QCD analyses using their data as input to the fits, e.g. ZEUS-JETS [10] or the recent H1PDF2012 [5]. An uniform approach was developed by $\mathrm{H} 1$ and ZEUS to fit the combined HERA data as the only input. The HERAPDF QCD fitting framework, which was developed, is based on QCDNUM for the NLO and NNLO QCD evolution. In the HERAPDF fits a variable-flavour-number scheme is used. The usage of data from HERA only, with well controlled systematic uncertainties, allows a $\Delta \chi^{2}=1$ criterium for the determination of the experimental errors of the fit to be used. The uncertainties of the PDFs comprise also variations of the model assumptions like $Q_{\min }^{2}, m_{c}, m_{b}$ and the uncertainty related to the variation of the form of the PDF parametrisations at the starting scale, $Q_{o}^{2}=1.9 \mathrm{GeV}^{2}$.

The set of parton distribution functions, HERAPDF1.0 [2], is obtained in the fit to the combined HERA I data. The preliminary combination of the HERA I and HERA II data is used in the HERAPDF1.5 fit [11]. In comparison to the published HERAPDF1.0 the HERA II high $Q^{2}$ data further constrain the high $x$ 


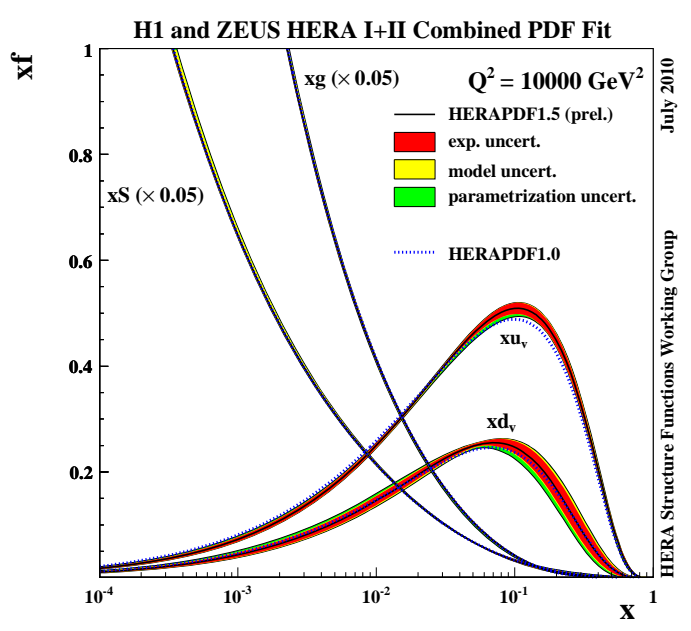

Figure 2: Parton distribution functions of HERAPDF1.5 [11] at the evolved scale of $Q^{2}=10000 \mathrm{GeV}^{2}$. The gluon and sea distributions are scaled by a factor 0.05 .

valence PDFs. The resulting PDFs have total uncertainties at the level of a few percent at low $x$. HERAPDF1.5 is the currently recommended PDFs set from HERA for usage at the LHC. In Figure 2 the HERAPDF1.5 parton distribution functions are shown at $Q^{2}=10000 \mathrm{GeV}^{2}$ corresponding to the LHC kinematic domain.

The impact of heavy flavor production data on the HERAPDF1.0 fit is studied by inclusion of the preliminary combined $F_{2}^{c c}$ data from HERA into the fit [12]. It was found that the $\chi^{2}$ optimum of the fits with different schemes for the treatment of heavy quarks corresponds to different values of the input charm mass parameter, $m_{c}$. Using optimal values of $m_{c}$, the prediction of $W$ and $Z$ production at the LHC is very stable with the spread of the predictions in different schemes within $1 \%$. In the HERAPDF1.6 [13] fit the HERA jet data are fitted together with the preliminary combined HERA I+II inclusive data. The inclusion of the jet data in the fit makes simultaneous determination of the PDFs and the strong coupling $\alpha_{s}\left(M_{Z}\right)$ possible. Finally, the inclusive HERA I and HERA II NC and CC data, the low proton beam energy data (2007), $F_{2}^{c c}$ and jet data from HERA are fitted together in the HERAPDF1.7 [14] fit.

The HERAFitter tool [15] has been developed by $\mathrm{H} 1$ and ZEUS as an open access code for the QCD PDF fits. The package is applicable to all types of data used in global PDF fits: inclusive DIS cross sections from HERA and fixed target experiments; heavy quark structure functions; jet production data in DIS, at the Tevatron and the LHC; Drell-Yan (including W, Z) cross sec- tions from fixed target experiments, the Tevatron and the LHC. The package is under continuous development providing a common set of tools for PDF fitting. The first results from the LHC obtained using HERAFitter were published by ATLAS [16]. In the ATLAS QCD fit to the $W^{ \pm}$and $Z$ rapidity distributions and the HERA inclusive data, the strange quark density at the starting scale is fitted as a free parameter. The fit clearly prefers unsuppressed strangeness in the proton contrary to fixed target neutrino di-muon data which favour a suppression of $\approx 50 \%$ compared to the down sea quark density.

\section{Summary}

The H1 and ZEUS experiments at HERA measured inclusive ep DIS cross sections over six orders of magnitude in $Q^{2}$ and $x$. Recently the HERA measurements were completed by publishing the NC and CC cross sections at high $Q^{2}$ from HERA II (H1) and the $e^{+} p$ NC cross section from the years 2006-2007 (ZEUS). The electromagnetic structure function $F_{2}$ is determined with an accuracy of about $1 \%$ in the best measured region. The proton structure functions $F_{L}$ and $x F_{3}^{\gamma Z}$ are measured as well. The polarisation effects in NC and $\mathrm{CC}$ are studied. This led to the first measurement of the parity violating structure function $F_{2}^{\gamma Z}$. The H1 and ZEUS data are combined in a preliminary form and used to determine PDFs. HERAPDF1.5 is the currently recommended PDFs set from HERA to be used at the LHC. The first results using HERAFitter, an open access tool for PDF fits, are obtained at the LHC by the ATLAS Collaboration.

\section{References}

[1] H1 Collab., DESY-12-063, submitted to EPJC.

[2] H1 and ZEUS Collab., F. D. Aaron et al., JHEP01, 109 (2010).

[3] ZEUS Collab., S. Chekanov et al., Eur. Phys. J., C61, 223 (2009); C62, 625 (2009); H. Abramowicz et al., Eur. Phys. J., C70, 945 (2010)

[4] H1 Collab., H1prelim-09-042, H1prelim-09-043.

[5] H1 Collab., DESY-12-107, accepted at JHEP.

[6] H1 Collab., DESY-12-145.

[7] H1 Collab., F. D. Aaron et al., Phys. Lett., B665, 139 (2008); H1 Collab., H1prelim-08-042; H1 Collab., F. D. Aaron et al., Eur. Phys. J., C71, 1579 (2011).

[8] ZEUS Collab., S. Chekanov et al., Phys. Lett., B682, 8 (2009).

[9] H1 and ZEUS Collab., H1prelim-10-141, ZEUS-prel-10-017.

[10] ZEUS Collab., S. Chekanov et al., Eur. Phys. J., C42, 1 (2005).

[11] H1 and ZEUS Collab., H1prelim-10-142, ZEUS-prel-10-018.

[12] H1 and ZEUS Collab., H1prelim-10-143, ZEUS-prel-10-019.

[13] H1 and ZEUS Collab., H1prelim-11-034, ZEUS-prel-11-001.

[14] H1 and ZEUS Collab., H1prelim-11-143, ZEUS-prel-11-010.

[15] http://projects.herforge.org/herafitter

[16] ATLAS Collab., G. Aad et al., Phys. Rev. Lett., 109, 012001 (2012). 